Gynäkologe 2018 $51: 8-10$

https://doi.org/10.1007/s00129-017-4179-z

(c) Springer Medizin Verlag GmbH, ein Teil von Springer Nature 2018

CrossMark

K. Diedrich ${ }^{1}$ für die Schriftleitung von Der Gynäkologe • S. Schumacher-Schmidt ${ }^{2}$

'Endokrinologie und Osteologie, amedes Facharzt-Zentrum für Kinderwunsch pränatale Medizin, Hamburg, Deutschland

${ }^{2}$ Springer Medizin Verlag GmbH, Heidelberg, Deutschland

\title{
50 Jahre Der Gynäkologe
}

\section{Zur Historie der Zeitschrift}

Liebe Leserinnen und Leser,

vor 50 Jahren wurde von den engagierten und hoch renommierten Frauenärzten Volker Friedberg, Otto Käser und Ernst Jürgen Plotz eine neue Zeitschrift zur ,Information und Fortbildung" (• Abb. 1) für Fachärzte der Gynäkologie und Geburtshilfe gegründet: Der Gynäkologe. Bereits im September 1968 erschien die erste Ausgabe zum Thema „Postoperative Komplikationen in der Gynäkologie“. Prof. Friedberg ließ es sich nicht nehmen, diese höchstselbst herauszugeben und zu redigieren.

Noch heute zeichnen Mitglieder des mittlerweile stark gewachsenen Herausgeberboards verantwortlich für die einzelnen Ausgaben und betreuen mit viel Engagement, Liebe zum Detail und nicht zuletzt exzellenter fachlicher Expertise die Leitthemen.

Erschien Der Gynäkologe anfangs noch vierteljährlich, wurde er im Jahr 1989 auf 6 und in 1996 auf 9 Ausgaben jährlich aufgestockt, um seit $1997 \mathrm{zu}$ einer monatlichen Erscheinungsweise $\mathrm{zu}$ wechseln. Vor 50 Jahren war die Zeitschrift noch schwarz-weiß gedruckt, nur einzelne Anzeigen erschienen in Farbe. Mittlerweile ist eine 4-farbige Aufmachung selbstverständlich.

\section{》) Von Beginn an war das Kon- zept der Schwerpunktthemen prägend}

Von Beginn an prägend war das Konzept der Schwerpunktthemen. Für jede Ausgabe wurden ein Schwerpunkt festgelegt, speziell zu diesem Thema mehrere Übersichtsbeiträge eingeladen und ver- fasst sowie durch eine „Einführung zum Thema" des jeweiligen Heftherausgebers kommentiert. Auch heute noch planen und koordinieren die Mitglieder des Boards Schwerpunktthemen und laden hochrangige Autoren ein, ihr Spezialgebiet in einer Übersicht zusammenzufassen. Abgehandelt werden in diesem Zuge aktuelle Leitlinien, Kongressthemen sowie praxisrelevante Bereiche der Gynäkologie und Geburtshilfe. Die erste „Einführung zum Thema“ zeigt • Abb. 2.

Neben den Leitthemen ist die CME (,continuing medical education")-Rubrik ein wichtiger Bestandteil geworden. Erstmalig erschienen Artikel unter dem Namen,Weiterbildung "im Jahr 1996, seit dem Jahr 2000 werden sie von der Ärztekammer zertifiziert. Teilnehmer können den zertifizierten Fragebogen online beantworten und Fortbildungspunkte sammeln. Seit 2016 erfolgt die CMEThemengestaltung nach einer 5-Jahres-Säulenplanung, die alle 4 Säulen der Frauenheilkunde gleichermaßen berücksichtigt.

\section{》) Inzwischen erscheinen alle neuen Artikel online-first und sind dann schon zitierfähig}

Wie die Zusammensetzung der Rubriken haben sich auch das Layout und das gesamte Erscheinungsbild der Zeitschrift verändert, sie wird auch weiterhin dynamisch bleiben. Dabei wurde und wird Rücksicht genommen auf das Lese- und Nutzerverhalten sowie auf die Ansprüche der Leser. Aber auch die Anforderungen und Wünsche von Autoren, Herausgebern und Verlag tragen dazu bei.
Gerade das Leseverhalten befindet sich im Wandel. Startete die Zeitschrift 1968 noch als reines „Print-Produkt“ als gedrucktes Heft - so gibt es heute selbstverständlich diverse Möglichkeiten der Online-Nutzung. Diese hat in den vergangenen Jahren enorm zugenommen. Auf der Online-Plattform https:// link.springer.com sind alle seit 1996 erschienenen Artikel als PDF- oder HTMLVersion in einem Volltextarchiv verfügbar. Alle neuen Artikel erscheinen noch vor dem gedruckten Heft online-first und sind dann bereits zitierfähig. Die Anzahl an Artikel-Downloads steigt jährlich und übertraf im Jahr 2016 erstmalig die 100.000er-Marke.

Auch die deutschsprachige MedizinPlattform https://www.springermedizin. de verzeichnet eine stetig wachsende Zahl an Seitenaufrufen. Dort sind ebenfalls alle Artikel als PDFs verfügbar, und seit etwa einem Jahr steht die Zeitschrift online auch als ePaper zur Verfügung.

Im Laufe dieses Jahres werden historische Artikel renommierter Autoren kommentiert und gedruckt werden. Prof. Hans Ludwig wird in einer Sonderausgabe im September über die 3 Gründer berichten. Diese Sonderausgabe wird sich den Meilensteinen des Fachs widmen und dadurch zahlreiche Themen einbinden. Freuen Sie sich außerdem auf den 62. DGGG(Deutsche Gesellschaft für Gynäkologie und Geburtshilfe)-Kongress in Berlin. Dort wird Ihnen wieder ein spannendes Heft präsentiert, das passend zum Kongress von der amtierenden Präsidentin Prof. Seelbach-Göbel und dem Kongresspräsidenten Prof. Wagner gestaltet wird.

In dieser Ausgabe kommentiert Prof. Vetter den im Gründungsjahr 1968 
„Der Gynakologe“ soll der Information und Fortbildung des Facharztes fur Frauenheilkunde und Geburtshilfe dienen Dies crscheint um so notwendiger, da die Forschung wahrend der vergangenen Jahre in unserem Fachgebiet viele neue Erkenntnisse gebracht hat, die in den Lehr- und Handbuchern nicht mit der notwendigen Aktualitat vermittelt werden konnen Somit sollen in dieser Zeitschrift vor allem neuere Ergebnisse der wissenschaftlichen Forschung und der modernen klinischen Praxis unseres Fachgebietes und der wichtigen Nachbargebiete (Padiatrie, Innere Medızın, Chirurgie usw ) dargestellt werden

Jedes Heft wird einem bestımmten 'Thema aus der Gynakologie, der Geburtshilfe, der Endoknnologie oder der Padiatrie gewidmet sein. Die Auswahl der Lextthemen eines jeden Heftes erfolgt nach der von den Herausgebern angenommenen Aktualitat, wobeı die Prophylaxe, Diagnostuk und Therapie der Erkrankungen in den Vordergrund geruckt werden sollen Von den Herausgebern werden dazu kompetente Autoren aufgefordert, die in Form von Ubersichten uber diese aktuellen Themen unscres Fachgebietes berichten sollen. Die Zeitschrift wird zunachst vierteljahrlich erscheinen.

\section{Der Gynäkologe}

Band 1, Heft 1

September 1968

\section{Einführung zum Thema}

Der Fortschratt in der Betreuung von operierten Patuenten kann wohl mit Recht als eine der wichtigsten Errungenschaften der Chururgie in den letzten Jahrzehnten bezerchnet werden. Dadurch war es moghch, nicht nur die Technk operativer Eingruffe zu verbessern und zu erwettern, sondern auch die Indikationsstellung zur Operation auf Personengruppen auszudehnen, die man wegen ihres Alters oder wegen thres Grundleidens vor emigen Jahrzehnten noch nicht operieren konnte. Mit zunehmendem Umfang des Eingriffs wachst aber auch die Gefahr des Auftretens postoperatıver Komplikationen. Obwohl unsere Kenntnisse uber die Stoffwechselveranderungen nach chirurgischen Eingriffen bei weitem noch necht vollstandig sind und dee Ausdeutungen noch manche Fehler aufweisen, besitzen wr heute doch zahlreiche Angaben, welche bei der taghchen Behandlung am Krankenbett eine wertvolle Hulfe ergeben.

Nach emer Operation steht die Erhaltung und Wiederherstellung vitaler Funktionen im Vordergrund, wie Atmung, Kreislauf und Nierenfunktion. Jede Form emer akuten Hypoxie fuhrt aber durch eme Schadigung der Kapillarmembran zum Gewebsodem. Von dem Grad und der Zeitdauer der Hypoxie hangt es ab, ob irreversible Organschaden auftreten, die durch ihren eigengesetzlichen Verlauf die Heilerfolge einer Therapie in Frage stellen und $z u$ emer Elementargefahrdung des Lebens fuhren. Auch eme Beeintrachtigung der Darmtatigkeit im Sinne enes paralytischen Ileus kann in der postoperativen Phase zu einem ernsten Problem werden.

Aus diesen genannten postoperativen Komplukationen ergibt sich elgentlich zwangslaufig, $d a \beta$ in der akuten Phase nach einer Operation eine Intensw -
Uberwachung und -Behandlung notwendrg $2 s t$, die gesonderte Aufwachraume oder Wachstationen erforderlich macht Dabei 1st die Zusammenarbett des Operateurs mit den Fachanaesthesisten unerlaßhch, da diese durch ihre uberwegende Ausbuldung in chirurgischen Kliniken bei bestımmten Komplukatıonen, wie z.B beim Schock oder beim akuten Herzstillstand, eine sehr viel großere Erfahrung besitzen und auch in der Intensivpflege in den letzten Jahren eine große Aktivitat entfaltet haben Andererserts ist es aber auch fur den Operateur unbedingt notwendig, sich standıg mit den Moglichkeiten der postoperativen Komplikationen vertraut zu machen, um dann vorbeugend emgreffen zu konnen Dabel ist ein fruhzeitsges Erkennen einer erhohten Operationsgefahrdung und eine ausreichende praoperative Vorbehandlung genau so wichtig wie eme exakte postoperative Nachbehandlung.

Der Zweck dieses Heftes mit dem Leitthema „Postoperatıve Komplkationen" ist es daher, einge der bekanntesten und haufigsten postoperatıven Storungen zu schildern, die oft den Erfolg auch emer sehr gut ausgefuhrten Operation zumachte machen konnen. Wenn man die Ursachen und die Pathophysiologie dieser Kompllkationen besser versteht, so konnen ihre Symptome durch eine gute klinische Beobachtung zusammen mit biochemischen Plasmaanalysen, welche in jedem Krankenhaus leicht durchfuhrbar sind, rechtzeitig erkannt und richtig behandelt werden. Die Erschemungsformen dieser Storungen und auch die Therapievorschlage wurden in diesem Heft von den Referenten bewußt veremfacht dargestellt, damit die wesenthchen Gesichtspunkte im Gedachtnis des Lesers haften
Abb. $1<$ Aims and Scope der Ausgabe $1 / 1968$ von Der Gynäkologe
Die Herausgeber
Abb. $2 \triangleleft$ „Einführung zum Thema" in Ausgabe 1/1968 
erschienenen Artikel von E. Glatthaar „Die traumatische Gefährdung des $\mathrm{Fe}$ tus“. Prof. Zimmermann und Prof. Vetter nehmen sich des Schwerpunkts „Kontroversen in der Pränataldiagnostik und ihre Konsequenzen“ an.

Die Schriftleitung und der Verlag freuen sich sehr, dass Sie, liebe Leserinnen und Leser, der Zeitschrift treu verbunden sind, und hoffen, Ihnen mit den Jubiläumsaktionen Freude zu bereiten.

Eine gute Lektüre mit den allerbesten Grüßen an Sie alle wünschen Ihnen

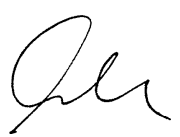

\section{K. Diedrich}

\section{St. Salvomale-Schmict}

\section{S. Schumacher-Schmidt}

\section{Korrespondenzadresse}

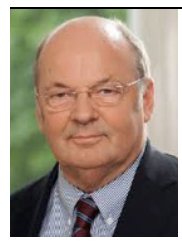

Prof. Dr. med. K. Diedrich

Endokrinologie und

Osteologie, amedes Facharzt-

Zentrum für Kinderwunsch

pränatale Medizin

Mönckebergstraße 10, 20095 Hamburg, Deutschland prof.diedrich@gmail.com

Interessenkonflikt. K. Diedrich und S. SchumacherSchmidt geben an, dass kein Interessenkonflikt besteht.

\section{Modellprojekt zur Prävention von sexuell übertragbaren Infektionen}

\section{Ärztliche Gesellschaft zur Gesundheitsförderung bietet Informationsstunden zu STI in Schulen an}

Es hat eine lange Tradition, dass Ärztinnen und Ärzte der Ärztlichen Gesellschaft zur Gesundheitsförderung e.V. (ÄGGF) Kinder, Jugendliche und junge Erwachsene in Schulen altersabhängig über Themen zur sexuellen und reproduktiven Gesundheit informieren.

Bisher standen sexuell übertragbare Infektionen (STI) weniger im Mittelpunkt der schulischen Sexualaufklärung. Jugendliche und junge Erwachsene wissen noch immer nur wenig über die Übertragungsrisiken, Schutzmöglichkeiten und Symptome von STI, obwohl sie teilweise sogar selbst von STI wie Chlamydien oder HPV betroffen sind.

Die ÄGGF nimmt das zum Anlass, mit Beginn des neuen Schuljahres bundesweit Informationsstunden zu STI in Schulen anzubieten. Das auf drei Jahre angelegte Modellprojekt wird von der Bundeszentrale für gesundheitliche Aufklärung (BZgA) und dem Verband der Privaten Krankenversicherung e. V. (PKV) gefördert.

Hierzu erklärt Dr. Heidrun Thaiss, Leiterin der BZgA: "Das Modellprojekt ergänzt die umfangreiche Arbeit der BZgA im Bereich der HIV/STI-Prävention in der Lebenswelt Schule. Wir halten es für dringend erforderlich, das Wissen zu den relevanten STI bereits im Jugendalter zu erhöhen und dadurch junge Menschen in ihrer Handlungskompetenz zu stärken. Der aufsuchende primärpräventive Ansatz der ,ärztlichen Informationsstunde' leistet hierzu einen wichtigen Beitrag."

Dr. Volker Leienbach, Direktor des Verbandes der Privaten Krankenversicherung e.V, ergänzt: "Gerade im Jugendalter beschäftigen sich junge Menschen stark mit dem Thema Sexualität. Es ist deshalb ein guter Zeitpunkt, um sie auch über die Gefahren von AIDS und anderen sexuell übertragbaren Krankheiten aufzuklären. Das versetzt sie in die Lage, sich verantwortungsbewusst zu verhalten und Risiken zu vermeiden, die sie unter Umständen ihr Leben lang bereuen könnten."
Die Ärztinnen und Ärzte der Ärztlichen Gesellschaft zur Gesundheitsförderung bestätigen ein hohes Interesse und ein Informationsbedürfnis bei den jungen Menschen zu STI. Diese Beobachtungen decken sich mit den Ergebnissen der Repräsentativerhebung "AIDS im öffentlichen Bewusstsein 2016", die die BZgA in regelmäßigen Abständen durchführt. Während HIV/AIDS einen hohen Bekanntheitsgrad in der Bevölkerung hat, sind andere für Deutschland relevante STI deutlich weniger bekannt.

Die Ärztinnen und Ärzte der ÄGGF sprechen mit den Jugendlichen in Mädchen- und Jungengruppen in vertraulicher Atmosphäre ab der 8. Klasse. Wichtige Themen sind dabei neben der Kenntnis verschiedener STI Übertragungsrisiken, Verhütungsmethoden und Schutzmaßnahmen (Safer Sex/Impfen), das Erkennen von Symptomen, die Kommunikation mit dem Partner bzw. der Partnerin sowie die Information über geeignete Beratungsund Behandlungsangebote.

Quelle: Bundeszentrale für gesundheitliche Aufklärung, www.bzga.de 